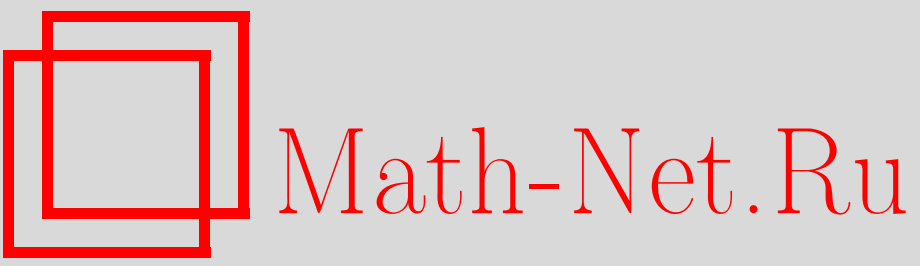

Л. А. Калякин, Асимптотическое решение задачи об авторезонансе, ТMФ, 2002, том 133, номер 3, 429-438

DOI: https://doi.org/10.4213/tmf409

Использование Общероссийского математического портала Math-Net.Ru подразумевает, что вы прочитали и согласны с пользовательским соглашением

http://www.mathnet.ru/rus/agreement

Параметры загрузки:

IP : 54.198 .64 .247

26 апреля 2023 г., 12:37:45 
ТЕОРЕТИЧЕСКАЯ

И МАТЕМАТИЧЕСКАЯ

ФИЗИКА

Том 133, № 3

декабрь, 2002

(C) 2002 г.

Л. А. Калякин ${ }^{*}$

\section{АСИМПТОТИЧЕСКОЕ РЕШЕНИЕ ЗАДАЧИ ОБ АВТОРЕЗОНАНСЕ}

Исследуется задача о вынужденных колебаниях вблизи устойчивого положения равновесия для двумерной нелинейной гамильтоновой системы уравнений. Заданная вынуждающая сила представляет собой быстро осциллирующие колебания с малой амплитудой и медленно меняющейся частотой. Выясняются условия, при которых фазовая траектория системы под влиянием такого возмущения уходит от начальной точки равновесия на расстояние порядка единицы. Исследование задачи проводится путем построения асимптотического решения с использованием малого амплитудного параметра. Решение представляется для не слишком малых времен вне начального пограничного слоя.

Ключевые слова: нелинейные колебания, резонанс, асимптотика, усреднение.

\section{1. ВВЕДЕНИЕ}

1.1. Постановка задачи. Рассмотрим систему двух обыкновенных дифференшиальных уравнений с малым параметром $\varepsilon$ :

$$
\frac{d \mathbf{u}}{d t}+\Omega(\mathbf{u})=\varepsilon \mathbf{F}\left(\frac{\Phi(\tau)}{\varepsilon} ; \tau\right), \quad \mathbf{u} \in \mathbb{R}^{2}, \quad t \in \mathbb{R}, \quad \tau=\varepsilon t, \quad 0<\varepsilon \ll 1 .
$$

Здесь $\Omega(\mathbf{u}), \mathbf{F}(\varphi ; \tau) \in \mathbb{R}^{2}$ - заданные гладкие (бесконечно дифференцируемые) векторфункции; $\mathbf{F}(\varphi ; \tau)$ является $2 \pi$-периодической функцией по быстрой переменной $\varphi=$ $\Phi(\tau) / \varepsilon$ с $\Phi(0)=0$. Переменная $\tau=\varepsilon t$ обычно называется медленным временем. Система предполагается гамильтоновой с голоморфным гамильтонианом $H(u, v), \mathbf{u}=(u, v)$. Считается, что невозмушенные уравнения имеют в начале координат устойчивое положение равновесия типа "центр" обшего положения; таким образом, $\Omega(\mathbf{0})=\mathbf{0}$, собственные значения матришы $\partial_{\mathbf{u}} \Omega(\mathbf{0})$ являются чисто мнимыми и не равны нулю. Начальные данные задаются в точке равновесия, либо вблизи нее:

$$
\left.\mathbf{u}(t ; \varepsilon)\right|_{t=0}=\varepsilon\left(\begin{array}{c}
\alpha_{1} \\
\alpha_{2}
\end{array}\right), \quad 0<\varepsilon \ll 1, \quad \alpha_{1}, \alpha_{2}=\text { const } .
$$

\footnotetext{
* Институт математики Уфимского научного центра РАН, Уфа, Россия. E-mail: klen@imat.rb.ru
} 
Мы ставим задачу о построении асимптотики решения $\mathbf{u}(t ; \varepsilon)$ при $\varepsilon \rightarrow 0$, пригодной на большом временно́м интервале $0<t \leqslant \mathcal{O}\left(\varepsilon^{-1}\right)$. Интерес представляет случай авторезонанса, когда амплитуда решения $|\mathbf{u}(t ; \varepsilon)|$ вырастает со временем до величины порядка единицы при том, что вынуждающая сила (накачка) остается малой: $\varepsilon \mathbf{F}(\varphi ; \tau)=\mathcal{O}(\varepsilon)^{1)}$. Данная работа посвяшена выяснению условий возникновения этого явления путем исследования асимптотического решения уравнения (1.1).

1.2. Уточнение исходных данных. Структура рассматриваемой точки равновесия подразумевает, что в ее окрестности невозмушенная система (при $\varepsilon \mathbf{F} \equiv \mathbf{0}$ ) имеет двухпараметрическое семейство периодических по $t$ решений $\mathbf{u}^{0}(t+\psi ; E), \psi, E=$ const. Считается, что точка равновесия соответствует нулевому значению параметра $E: \mathbf{u}^{0}(t+\psi ; 0) \equiv \mathbf{0}$. Этот параметр, как первый интеграл системы, отождествляется с энергией: $E=H\left(u^{0}, v^{0}\right)$, которая может принимать значения на некотором промежутке $E \in\left(0, e_{0}\right) \subset \mathbb{R}$. Период невозмушенных (собственных) колебаний $T=T(E)$ и частота $\omega=2 \pi / T(E)$ в нелинейной системе, как правило, зависят от энергии $E$. В дальнейшем считается, что зависимость от $E$ сушественная, так что $T^{\prime}(E) \neq 0, \omega^{\prime}(E) \neq 0$.

1.3. Известные результаты для похожих задач. Возмушение периодических движений с давних пор представляет собой одну из популярных задач нелинейной механики и теории колебаний. В качестве возмушений чаще всего рассматриваются функции $\mathbf{F}(\mathbf{u} ; \tau)$, зависящие от искомого движения $\mathbf{u}=\mathbf{u}(t ; \varepsilon)$ и, может быть, от медленного времени $\tau=\varepsilon t$. Известно, что при таких возмущениях главный член асимптотики описывается решением невозмущенной системы $\mathbf{u}(t ; \varepsilon) \approx \mathbf{u}^{0}\left(\left[\varepsilon^{-1} S(\tau)+\Psi(\tau)\right] / \omega(E) ; E(\tau)\right)$, в котором медленно меняются как фазовые функции $S, \Psi(\tau)$, так и энергия $E(\tau)$. При этом энергия изменяется на конечную величину порядка $\mathcal{O}(1)$ за большой промежуток времени $0<t \leqslant \mathcal{O}\left(\varepsilon^{-1}\right)$, если начальная точка находится вдали от положения равновесия (см. [1]-[3]). Если начальная точка берется вблизи устойчивого положения равновесия на расстоянии $\mathcal{O}(\varepsilon)$, то решение остается в малой окрестности порядка $\mathcal{O}(\varepsilon)$ в течение долгого времени $0<t \leqslant \mathcal{O}\left(\varepsilon^{-1}\right)[4]$.

Задачи с возмущениями, которые явно зависят от быстрого времени, $\mathbf{F}(t ; \tau)$, обычно связываются с изучением нелинейного резонанса и синхронизации колебаний. В случае периодических по $t$ возмушений здесь возможен резонанс со значительным ростом амплитуды вынужденных колебаний. В частности, известно, что для решений, начинающихся из положения равновесия, амплитуда может вырасти до величины порядка $\mathcal{O}\left(\varepsilon^{1 / 2}\right)$. При фиксированной частоте накачки больший рост невозможен, поскольку нарушается резонансное условие из-за изменения собственной частоты системы с ростом

\footnotetext{
1) Значительный по сравнению с вынуждающей силой рост амплитуды вынужденных колебаний обычно связывается с резонансом. Простейшая математическая модель такого явления представляется линейным уравнением гармонического осциллятора $u^{\prime \prime}+\omega^{2} u=\cos \nu t$. В случае, когда накачка осциллирует с собственной частотой: $\omega^{2}=\nu^{2}$, амплитуда вынужденных колебаний неограниченно растет: $u=(2 t / \nu) \sin \nu t$. Подобное явление в случае нелинейных систем связывается с понятием авторезонанса.
} 
энергии [2], [3]. Иная ситуация складывается при медленном изменении частоты накачки. Амплитуда вынужденных колебаний здесь может достигать величины порлдка единицы. Первоначально идея использования такой накачки была выдвинута физиками и разрабатьвалась в связи с созданием ускорителей [5]. В последние годы найдены другие области нелинейных явлений, в которых обнаруживаются похожие эффекты [6], [7] . Были проведены численные эксперименты и аналитические исследования ряда моделей такого явления, названного авторезонансом [7]. Все аналитические исследования в этом направлении так или иначе используют асимптотические приближения. Однако последовательный асимптотический анализ задач об авторезонансе до сих пор не проводился даже для простых моделей, и условия авторезонанса не были отчетливо сформулированы.

1.4. Основной результат. Для рассматриваемой нами задачи $(1.1),(1.2)$ основной результат выглядит довольно естественно. Вынужденные колебания в главном члене асимптотики описываются решением невозмушенной системы

$$
\mathbf{u}(t ; \varepsilon)=\mathbf{u}^{0}\left(\frac{\varepsilon^{-1} \Phi(\tau)+\Psi(\tau)}{\omega} ; E(\tau)\right)+\mathcal{O}(\varepsilon), \quad \varepsilon \rightarrow 0
$$

с медленно деформируюшимися параметрами $\Psi(\tau), E(\tau), \omega(E(\tau)), \tau=\varepsilon t$. Деформация энергии $E(\tau)$ определяется из резонансного требования совпадения собственной и вынуждающей частот

$$
\omega(E)=\Phi^{\prime}(\tau), \quad E(0)=0 .
$$

С двиг фазы $\Psi$ находится из нелинейного уравнения, которое является следствием секулярного условия для первой поправки. Это уравнение выписывается с использованием среднего от кососкалярного произведения двух векторов:

$$
\frac{1}{T(E)} \int_{0}^{T}\left[\partial_{\sigma} \mathbf{u}^{0}(\sigma ; E), \mathbf{F}(\sigma \omega(E)-\Psi ; \tau)\right] d \sigma=\frac{\Phi^{\prime \prime}(\tau)}{\omega^{\prime}(E)} .
$$

Требования разрешимости этой пары уравнений относительно $E, \Psi$ представляют собой основные условия на исходные данные, при которых только и существует асимптотическое решение в форме (1.3). Выполнения одного резонансного условия (1.4) недостаточно. Исследования ряда моделей [7] выявили принципиальные отличия нелинейной системы от линейной: было обнаружено, что для возникновения авторезонанса необходимо превышение амплитуды накачки над некоторым пороговым значением. Ограничения порогового характера обнаруживаются в уравнении (1.5) для сдвига фазы $\Psi$. Дальнейшее изложение посвяшено выводу уравнений (1.4), (1.5) и конструкции полного асимптотического решения при $\varepsilon \rightarrow 0$ с указанием области применимости по времени. 


\section{2. БЫСТРЫЕ И МЕДЛЕННЫЕ ДВИЖЕНИЯ}

2.1. Анзац. В этом разделе мы будем следовать стандартной процедуре построения асимптотического решения в форме анзаца Крылова-Боголюбова-Кузмака [1]. В основе обсуждаемого подхода лежит простое соображение, что главный член асимптотики можно представить в виде решения невозмушенной системы $\mathbf{u}(t ; \varepsilon)=\mathbf{u}^{0}(\sigma ; E)+\mathcal{O}(\varepsilon)$ при подходяшем выборе фазы $\sigma=\left[\varepsilon^{-1} \Phi(\tau)+\Psi(\tau)\right] / \omega(E)$ и при подходяшей деформации параметра $E=E(\tau)$. Функции $E(\tau), \Psi(\tau)$ зависят от медленного времени $\tau=\varepsilon t$, и их требуется найти.

В новых переменных выражение для правой части уравнений (1.1) можно привести к виду функции $\mathbf{F}(\varphi ; \tau)=\mathbf{F}(\omega(E) \sigma-\Psi ; \tau), \quad T$-периодической по переменной $\sigma$. При таком представлении обнаруживается, помимо зависимости от быстрой переменной $\sigma$, зависимость от неопределенных пока функций $E(\tau), \Psi(\tau)$, а также явная зависимость от медленного времени $\tau$. Решение возмушенной задачи ишется в подобной форме, как вектор-функция четырех независимых переменных $\mathbf{u}(t ; \varepsilon)=\mathbf{U}(\sigma, E, \Psi, \tau ; \varepsilon)$ с периодической зависимостью по быстрой переменной $\sigma$. Полное формальное асимптотическое решение (ФАР) строится в виде ряда

$$
\mathbf{u}(t ; \varepsilon)=\sum_{n=0}^{\infty} \varepsilon^{n} \mathbf{U}_{n}(\sigma, E, \Psi, \tau), \quad \varepsilon \rightarrow 0 ; \quad \sigma=\frac{\varepsilon^{-1} \Phi(\tau)+\Psi}{\omega(E)}
$$

Коэффициенты ряда, а также функции $E(\tau), \Psi(\tau)$ определяются из рекуррентной системы задач. Так, для ряда хорошо изученных задач известно, что функции медленного времени $E(\tau), \Psi(\tau)$ определяются из дифференциальных уравнений, которые иногда называются усредненными уравнениями [1], [2]. Для задачи об авторезонансе здесь возникают принципиальные отличия: эти функции определяются из функциональных уравнений.

2.2. Разделение движений. Процедура разделения задач для быстрых и медленных переменных основана на анализе системы, которая получается для вектор-функции $\mathbf{U}(\sigma, E, \Psi, \tau ; \varepsilon)$. В уравнениях содержатся производные $E^{\prime}, \Psi^{\prime}$ в виде коэффициентов, которые остаются неопределенными, пока не определены сами функции $E(\tau), \Psi(\tau)$. Они определяются при вычислении полной производной по времени:

$$
\frac{d \mathbf{U}}{d t}=\frac{\Phi^{\prime}}{\omega} \partial_{\sigma} \mathbf{U}+\varepsilon E^{\prime}\left[\partial_{E} \mathbf{U}-\sigma \frac{\omega^{\prime}}{\omega} \partial_{\sigma} \mathbf{U}\right]+\varepsilon\left[\Psi^{\prime}\left(\partial_{\sigma} \mathbf{U}+\partial_{\Psi} \mathbf{U}\right)+\partial_{\tau} \mathbf{U}\right]
$$

В последнем выражении содержится пара непериодических по $\sigma$ слагаемых, которые, однако, в сумме дают периодическую функцию. В частности, ввиду зависимости периода от параметра $E$ непериодической оказывается частная производная $\partial_{E} \mathbf{U}$. Такие слагаемые удобно объединить, введя так называемую усеченную производную $\hat{\partial}_{E} \mathbf{U}=$ $\partial_{E} \mathbf{U}-\sigma\left(\omega^{\prime} / \omega\right) \partial_{\sigma} \mathbf{U}$, для которой сохраняется свойство периодичности. В разложении 
Фурье такая производная соответствует дифференцированию по $E$ только коэффициентов ряда. При использовании усеченной производной исходные уравнения принимают вид

$$
\frac{\Phi^{\prime}}{\omega(E)} \partial_{\sigma} \mathbf{U}+\Omega(\mathbf{U})=\varepsilon \mathbf{F}-\varepsilon\left[E^{\prime} \hat{\partial}_{E}+\Psi^{\prime}\left(\partial_{\sigma} \mathbf{U}+\partial_{\Psi} \mathbf{U}\right)+\varepsilon \partial_{\tau}\right] \mathbf{U}
$$

\section{3. РЕКУРРЕНТНАЯ СИСТЕМА ОТНОСИТЕЛЬНО БЫСТРОЙ ПЕРЕМЕННОЙ}

Цель данного раздела - выписать рекуррентную систему на коэффициенты $\Phi$ АР (2.1) и сформулировать секулярные условия, при которых этот ряд будет асимптотическим при $\varepsilon \rightarrow 0$ равномерно по быстрой переменной $\sigma \in \mathbb{R}$.

В качестве главного члена ФАР берется невозмушенное решение: $\mathrm{U}_{0}=\mathbf{u}^{0}(\sigma ; E)$. В таком случае множитель в левой части уравнения (2.2) должен быть равен единице: $\Phi^{\prime} / \omega(E)=1$, что соответствует требованию (1.4). Поправки следующих порядков находятся из неоднородных линеаризованных уравнений:

$$
\partial_{\sigma} \mathbf{U}_{n}+\partial_{u} \Omega\left(\mathbf{u}^{0}\right) \mathbf{U}_{n}=\mathbf{G}_{n}(\sigma, E, \Psi, \tau),
$$

где правые части определяются через предыдущие приближения с участием коэффициентов Тейлора вектор-функции $\Omega(\mathbf{u})$ в точке $\mathbf{u}=\mathbf{u}^{0}$. Например, на первых двух шагах имеем

$$
\begin{gathered}
\mathbf{G}_{1}=\mathbf{F}-\left[E^{\prime} \hat{\partial}_{E}+\Psi^{\prime} \partial_{\sigma}\right] \mathbf{u}^{0} \\
\mathbf{G}_{2}=-\frac{1}{2} \partial_{u}^{2} \Omega\left(\mathbf{u}^{0}\right) \mathbf{U}_{1} \mathbf{U}_{1}-\left[E^{\prime} \hat{\partial}_{E}+\Psi^{\prime}\left(\partial_{\sigma}+\partial_{\Psi}\right)+\partial_{\tau}\right] \mathbf{U}_{1}
\end{gathered}
$$

Для фундаментальной системы решений однородных уравнений удобно использовать пару периодических вектор-функций, составленных из производных: $\mathbf{V}_{0}(\sigma ; E)=$ $\hat{\partial}_{E} \mathbf{u}^{0}(\sigma ; E), \mathbf{V}_{1}(\sigma ; E)=\partial_{\sigma} \mathbf{u}^{0}(\sigma ; E)$. При этом одним из решений является $\mathbf{V}_{1}$, а второе выписывается через комбинацию $\mathbf{V}_{0}$ и $\mathbf{V}_{1}: \mathbf{V}_{2}=\mathbf{V}_{0}+\left(\omega^{\prime} / \omega\right) \sigma \mathbf{V}_{1}$, и не будет периодическим, если $\omega^{\prime}(E) \neq 0$. Вронскиан выражается через кососкалярное произведение, $W=\left[\mathbf{V}_{1}, \mathbf{V}_{2}\right]$, и равен единице, $W=1$.

На каждом шаге решение выписывается через интегралы от кососкалярных произведений базисных решений с правой частью:

$$
\begin{aligned}
\mathbf{U}_{n}= & +\mathbf{V}_{0} \int_{0}^{\sigma}\left[\mathbf{V}_{1}, \mathbf{G}_{n}\right] d \sigma+\mathbf{V}_{1} \int_{0}^{\sigma}\left(\left[\mathbf{G}_{n}, \mathbf{V}_{0}\right]+\frac{\omega^{\prime}}{\omega}\left(C_{n}+\int_{0}^{\sigma}\left[\mathbf{V}_{1}, \mathbf{G}_{n}\right] d \sigma\right)\right) d \sigma+ \\
& +C_{n} \mathbf{V}_{0}+D_{n} \mathbf{V}_{1} .
\end{aligned}
$$

Дополнительные слагаемые с произвольными функциями медленных переменных $C_{n}(E, \Psi, \tau), D_{n}(E, \Psi, \tau)$ соответствуют структуре обшего решения.

В обшем случае решение не будет периодическим. В формуле (3.2) нетрудно усмотреть наличие членов, которые растут линейно $\mathcal{O}(\sigma)$ и квадратично $\mathcal{O}\left(\sigma^{2}\right)$ при $\sigma \rightarrow \infty$.

4 Теоретическая и математическая физика, т. 133, № 3, 2002 г. 
Исключение таких секулярных слагаемых приводит к двум условиям на правую часть уравнения. При их выполнении решение оказывается периодическим. Ограничения на правые части $\mathbf{G}_{n}$ формулируются в виде условий ортогональности:

$$
\left\langle\left[\mathbf{V}_{1}, \mathbf{G}_{n}\right]\right\rangle=0, \quad \frac{\omega^{\prime}}{\omega} C_{n}+\left\langle\left[\mathbf{G}_{n}, \mathbf{V}_{0}\right]+\frac{\omega^{\prime}}{\omega} \int_{0}^{\sigma}\left[\mathbf{V}_{1}, \mathbf{G}_{n}\right] d \sigma\right\rangle=0
$$

Здесь и далее угловые скобки используются для обозначения среднего по периоду. В частности, на первом шаге эти два требования выглядят следуюшим образом:

$$
E^{\prime}(\tau)=\left\langle\mathbf{V}_{1}, \mathbf{F}\right\rangle, \quad \frac{\omega^{\prime}}{\omega} C_{1}=\Psi^{\prime}-\left\langle\mathbf{F}, \mathbf{V}_{0}\right\rangle+\frac{\omega^{\prime}}{\omega}\left\langle\int_{0}^{\sigma}\left(E^{\prime}-\left[\mathbf{V}_{1}, \mathbf{F}\right]\right) d \sigma\right\rangle
$$

\section{4. МЕДЛЕННАЯ ДЕФОРМАЦИЯ ГЛАВНОГО ЧЛЕНА ФАР}

На основе анализа главного члена $\Phi$ АР и первой поправки получаются три уравнения, приведенные в (1.4), (3.4). Из условия (1.4) определяется медленная деформация энергии $E=E(\tau)$. Корней уравнения может быть много; нужный корень выделяется начальным условием $E(0)=0$. Требование разрешимости такой задачи определяет класс вынуждаюших частот $\Phi^{\prime}$, при которых только и возможен авторезонанс. Приведем условия, которые представляются более доступными для проверки, чем резонансное условие в общей форме.

Tеорема 1. Пусть $\omega^{\prime}(0) \neq 0$. Уравнение (1.4) с условием $E(0)=0$ имеет единственное решение $E=E(\tau)$ на некотором промехутке $\tau \in\left[0, \tau_{0}\right]$ тогда и только тогда, когда частота накачки в начальный момент совпадает с собственной: $\Phi^{\prime}(0)=\omega(0)$, и совпадают направления изменения частот: $\operatorname{sgn} \Phi^{\prime \prime}(\tau)=\operatorname{sgn} \omega^{\prime}(0)$, $\tau>0$.

Первое из требований (3.4) дает уравнение для сдвига фазы $\Psi(\tau)$. С учетом уравнения (1.4) оно записывается в форме (1.5). Это уравнение можно несколько преобразовать:

$$
-\frac{1}{2 \pi} \int_{0}^{2 \pi}\left[\mathbf{u}^{0}\left(\frac{s}{\omega(E)} ; E\right), \partial_{s} \mathbf{F}(s-\Psi ; \tau)\right] d s=\frac{\Phi^{\prime \prime}(\tau)}{\Phi^{\prime}(\tau) \omega^{\prime}(E)} .
$$

Здесь в качестве $E=E(\tau)$ берется решение уравнения (1.4). Нетрудно установить условия его разрешимости в обшем случае.

Теорема 2. Пусть $\omega^{\prime}(E) \neq 0$ и уравнение (1.4) разрешимо на отрезке $\tau \in\left[0, \tau_{0}\right]$. Тогда существует пара функций $M_{-}(\tau), M_{+}(\tau)$ таких, что уравнение (1.5) разрешимо тогда и только тогда, когда правая часть соотношения (4.1) удовлетворяет неравенствам

$$
M_{-}(\tau) \leqslant \frac{\Phi^{\prime \prime}(\tau)}{\Phi^{\prime}(\tau) \omega^{\prime}(E(\tau))} \leqslant M_{+}(\tau) .
$$


При выполнении строгих неравенств решение будет неединственно.

В качестве функций $M_{ \pm}(\tau)$ берутся максимум и минимум по $\Psi$ функции из левой части (4.1), они полностью определяются возмушением $\mathbf{F}(s ; \tau)$. В конечном счете требование разрешимости в форме (4.2) представляет собой условия на исходные данные $\mathbf{F}(s ; \tau)$, $\Phi(\tau)$. Если уточнить класс возмушений $\mathbf{F}(s ; \tau)$, то условия разрешимости можно свести к более понятной форме. Так, если вектор накачки имеет одну гармонику:

$$
\mathbf{F}(\varphi ; \tau)=\left(\begin{array}{c}
\alpha \\
\beta
\end{array}\right) \cos \varphi, \quad \alpha=\alpha(\tau), \quad \beta=\beta(\tau),
$$

то уравнение для $\Psi$ можно свести к тригонометрическому уравнению, в котором участвуют два коэффициента Фурье от комбинации компонент $u_{0}=u_{0}(s / \omega(E), E), \quad v_{0}=$ $v_{0}(s / \omega(E), E)$ невозмушенного решения

$$
a(\tau)=\frac{1}{2 \pi} \int_{0}^{2 \pi}\left(\beta u_{0}-\alpha v_{0}\right) \sin s d s, \quad b(\tau)=\frac{1}{2 \pi} \int_{0}^{2 \pi}\left(\beta u_{0}-\alpha v_{0}\right) \cos s d s .
$$

Эти коэффишиенты известны постольку, поскольку известны компоненты невозмушенного решения $\left(u_{0}(\sigma, E), v_{0}(\sigma, E)\right)$ и энергия $E(\tau)$.

Теорема 3. Пусть правая часть в исходной задаче (1.1) задана в форме (4.3) $u \operatorname{sgn} \Phi^{\prime \prime}(\tau)=\operatorname{sgn} \omega^{\prime}(0)$. Уравнение (1.5) разрешимо тогда и только тогда, когда амплитуда и фаза накачки связаны неравенством

$$
\left[a^{2}(\tau)+b^{2}(\tau)\right]^{1 / 2} \geqslant \frac{\Phi^{\prime \prime}(\tau)}{\left|\Phi^{\prime}(\tau)\right| \omega^{\prime}(E(\tau))} .
$$

Существуют два и только два решения $\Psi=\Psi_{ \pm}(\tau)$. Решения различны $\Psi_{+}(\tau) \neq$ $\Psi_{-}(\tau)$, если неравенство (4.4) строгое.

Вывод. Полученные в вышеприведенных теоремах условия разрешимости уравнений (1.4), (1.5) можно интерпретировать как условия возникновения авторезонанса. Эти условия состоят из двух частей. Во-первых, требуется правильный выбор направления деформации частоты накачки в соответствии с изменением собственной частоты. Во-вторых, требуется достаточно большая величина отношения амплитуды накачки к скорости изменения частоты.

\section{5. МЕДЛЕННЫЕ ДЕФОРМАЦИИ ПОПРАВОК}

В старших поправках $\Phi$ AP $\mathbf{U}_{n}(\sigma, E, \Psi, \tau), n \geqslant 1$, зависимость от медленного времени проявляется как в явной форме, так и через функции $E(\tau), \Psi(\tau)$. Явная зависимость от $\tau$ находится при решении быстрых уравнений (3.1) с учетом подстановки в правые части выражений для производных $E^{\prime}(\tau), \Psi^{\prime}(\tau)$. Выражения для самих функций $E$, $\Psi$ на этом этапе можно не подставлять. В каждой поправке имеется пара медленных функций $C_{n}(E, \Psi, \tau), D_{n}(E, \Psi, \tau)$, которые находятся из секулярных условий (3.3). Все 
уравнения для $C_{n}, D_{n}$ являются линейными и решаются тривиально. Наиболее просто получаются выражения для $C_{n}$ :

$$
C_{n}(E, \Psi, \tau)=\frac{\omega}{\omega^{\prime}}\left\langle\mathbf{G}_{n}, \mathbf{V}_{0}\right\rangle+\left\langle\int_{0}^{\sigma}\left[\mathbf{V}_{1}, \mathbf{G}_{n}\right] d \sigma\right\rangle
$$

Однако прежде чем воспользоваться этой формулой при $n>1$, следует ликвидировать неопределенность в коэффициенте $D_{n-1}$, который входит в $\mathbf{G}_{n}$ посредством предыдущего приближения $\mathbf{U}_{n-1}$. Для этого используется секулярное условие $\left\langle\left[\mathbf{V}_{1}, \mathbf{G}_{n}\right]\right\rangle=0$. Это условие дает уравнение для $D_{n-1}$, которое выглядит столь же тривиально:

$$
-\omega \partial_{\Psi}\left\langle\left[\mathbf{V}_{1}, \mathbf{F}\right]\right\rangle D_{n-1}=g_{n-1}(E, \Psi, \tau)
$$

Правая часть $g_{n-1}$ здесь вычисляется через известные функции и не содержит неопределенностей. Выражение для коэффициента в левой части получено с учетом свойств первого приближения $\mathbf{U}_{1}$ и специфики матрицы линеаризованной системы (3.1). В общей ситуации этот коэффициент не обращается в нуль и имеет место

ТЕОРЕМА 4. Если для сдвига фазы $\Psi$ в главном члене ФАР выбран простой корень уравнения (1.5), то все коэффициенты $C_{n}, D_{n}$ в стариих поправках ФАР определяются однозначно из секулярных условий (3.3).

ВЫвод. ФАР в форме (2.1) строится однозначно с коэффициентами $\mathbf{U}_{n}(\sigma, E, \Psi, \tau)$, периодическими по быстрой переменной $\sigma$. Эти коэффициенты будут гладкими вектор-функциями для тех значений $E, \Psi, \tau$, для которых являются гладкими невозмущенное решение $\mathbf{u}^{0}(\sigma, E)$ и выбранные простые корни уравнений $(1.4),(1.5)$.

Требование гладкости (бесконечной дифференцируемости) связано с присутствием производных по $E, \Psi, \tau$ в правых частях рассмотренной выше рекуррентной системы уравнений.

\section{6. АСИМПТОТИКА ПО МАЛОЙ ЭНЕРГИИ}

При построении $\Phi А Р$ в форме (2.1) использовались лишь дифференциальные уравнения (1.1); выполнение начальных условий (1.2) при $\tau=0$ не гарантируется. На самом деле ситуация с начальным значением для $\Phi$ АР оказывается сложнее. Коэффициенты $\Phi$ AP $\mathbf{U}_{n}(\sigma, \tau)=\mathbf{U}_{n}(\sigma, E(\tau), \Psi(\tau), \tau)$, как сложные функции от $\tau$, имеют особенности при $\tau \rightarrow 0$. Чтобы вскрыть структуру этих особенностей, требуется асимптотика по малой энергии (при $E \rightarrow 0$ ) для всех используемых функций. Такая асимптотика извлекается из интегрального представления для невозмушенного решения, которое получается при интегрировании одного из уравнений с учетом закона сохранения $H(u, v)=E$. Приведем результаты для случая, когда гамильтониан не содержит линейной зависимости по одной из переменных. 
ТЕОрема 5. Пусть гамильтониан $H(u, v)$ - гладкая функиия, обладающая свойством $H_{v}(u, 0) \equiv 0 \quad \forall$ в окрестности рассматриваемого иентра общего положения. Тогда период колебаний $T=T(E)$ и частота $\omega=\omega(E)$ невозмущенных колебаний являются гладкими функииями әнергии $E$ u $T(0) \neq 0, \omega(0) \neq 0$.

СлЕДСТВИЕ 1. Простые корни $E(\tau), \Psi(\tau)$ уравнений $(1.4),(1.5)$ являются гладкими функииями по $\tau \in\left[0, \tau_{0}\right]$. Энергия имеет асимптотику $E(\tau)=E_{2} \tau^{2}+\mathcal{O}\left(\tau^{3}\right)$, $\tau \rightarrow 0$, с коэффициентом $E_{2}=\Phi^{\prime \prime \prime}(0) / \omega^{\prime}(0)$.

Источником особенностей в конечном счете оказывается негладкость по энергии невозмушенного решения $\mathbf{u}^{0}(\sigma ; E)=\mathcal{O}(\sqrt{2 E}), E \rightarrow 0$. Указание на такую негладкость можно усмотреть в законе сохранения.

ТЕОРема 6. Пусть выполнены условия теоремы 5. Тогда невозмущенное решение $\mathbf{u}(\sigma ; E)$ имеет разложсение в виде асимптотического ряда:

$$
\mathbf{u}^{0}(\sigma ; E)=\sqrt{2 E} \sum_{n=0}^{\infty} E^{n / 2} \mathbf{u}_{n}^{0}(\sigma \omega(E)), \quad E \rightarrow 0,
$$

равномерно для всех $\sigma \in \mathbb{R}$. Коэффициентьи $\mathbf{u}_{n}^{0}(s)$ представляют собой $2 \pi$-периодические вектор-функции своего аргумента.

СЛЕДСТВИЕ 2. Главный член в асимптотике (6.1) представляется в виде

$$
\mathbf{u}(\sigma ; E)=\sqrt{2 E}\left(\begin{array}{c}
\cos (\sigma \omega(E)) \\
-\sin (\sigma \omega(E))
\end{array}\right)+\mathcal{O}(E), \quad E \rightarrow 0 .
$$

Из последнего соотношения видно, что производные невозмушенного решения по $E$ имеют особенности в точке $E=0$. Такие производные входят в правые части рекуррентной системы задач (3.1). Поэтому получаемые решения $\mathbf{U}_{n}(\sigma, E, \Psi, \tau)$ будут иметь особенности при $E \rightarrow 0$.

ТЕОРема 7. Пусть выполнены условия теоремы 5 и в качестве $E=E(\tau), \quad \Psi=$ $\Psi(\tau), \quad \tau \in\left[0, \tau_{0}\right]$, выбраны простые корни уравнений (1.4), (1.5). Тогда коэффиииенты ФАР (2.1) определяются однозначно в классе функиий, периодических по бъстрой переменной, и имеют асимптотическое разложение

$$
\mathbf{U}_{n}(\sigma, E, \Psi, \tau)=E^{(1-3 n) / 2} \sum_{j=0}^{\infty} E^{j / 2} \mathbf{u}_{n, j}(\sigma, \Psi, \tau), \quad E \rightarrow 0,
$$

равномерное для всех рассматриваемых значений $\sigma, \Psi, \tau$. Коэффициенты этой асимптотики - гладкие функции. В ситуации общего положсния главный член отличен от нуля, так что $\mathbf{U}_{n}(\sigma, E, \Psi, \tau)=\mathcal{O}\left(E^{(1-3 n) / 2}\right)$.

Особенности по энергии $E$ переходят в особенности по медленному времени ввиду асимптотики $E(\tau)=\mathcal{O}\left(\tau^{2}\right), \tau \rightarrow 0$. Из-за этих особенностей ряд (2.1) перестает быть асимптотическим при малых $\tau$. 
СлЕДСтвИЕ 3. Ряд (2.1) представляет асимптотическое решение уравнения (1.1) лишь для не слишком малых времен: $\varepsilon t=\tau \gg \varepsilon^{(1 / 3)}$.

Область малых времен представляет собой аналог пограничного слоя, в котором асимптотическое решение строится другим способом, указанным в работе [8]. Конструкция в пограничном слое необходима для однозначной идентификации сдвига фазы $\Psi$ с использованием метода согласования [9].

Благодарности. Работа выполнена при поддержке РФФИ (грант № 00-01-00663, грант "Научные школы" № 00-15-96038) и INTAS (грант № 99-1068).

\section{Список литературы}

[1] Н. М. Крылов, Н. Н. Боголюбов. Исследование продольной устойчивости аэроплана. М.-Л.: Гос. авиац. и автотракт. изд-во, 1932; Г. Е. Кузмак. ПММ. 1959. Т. 23. № 3. С. 515; F. J. Bourland, R. Haberman. SIAM J. Appl. Math. 1988. V. 48. P. 737.

[2] Н. Н. Моисеев. Асимптотические методы в нелинейной механике. М.: Наука, 1981.

[3] Г. М. Заславский, Р. З. Сагдеев. Введение в нелинейную физику. От маятника до турбулентности и хаоса. М.: Наука, 1988.

[4] Н.Н. Боголюбов, Ю.А. Митропольский. Асимптотические методы в теории нелинейных колебаний. М.: Наука, 1974.

[5] В. И. Векслер. ДАН СССР. 1944. Т. 43. С. 346; С. 393; А. А. Андронов, Г. А. Горелик. ДАН СССР. 1945. Т. 49. С. 664; E. M. MacMillan. Phys. Rev. 1945. V. 68. P. 143.

[6] K.S. Golovanivsky. Phys. Scripta. 1980. V. 22. P. 126; К. С. Голованивский. Физика плазмы. 1985. Т. 11. № 3. C. 295.

[7] I. Aranson, B. Meersoh, T. Tajima. Phys. Rev. A. 1992. V. 45. P. 7505; L. Friedland. Phys. Rev. E. 1997. V. 55. P. 1929; 2000. V. 61. P. 3732; L. Friedland, A. G. Shagalov. Phys. Rev. Lett. 1998. V. 81. P. 4307.

[8] Л. А. Калякин. Докл. РАН. 2001. Т. 378. С. 594.

[9] А. М. Ильин. Согласование асимптотических разложений решений краевых задач. М.: Наука, 1989. 